\title{
Review on Printed Log Periodic and Yagi MSA
}

\author{
Sudesh R. Agrawal \\ PG student, Department of \\ EXTC \\ DJ Sanghvi College of \\ Engineering \\ Vile Parle (W) Mumbai, India
}

\author{
Kshitij A. Lele \\ PG student, Department of \\ EXTC \\ DJ Sanghvi College of \\ Engineering \\ Vile Parle (W) Mumbai, India
}

\author{
Amit A. Deshmukh \\ Professor and Head, \\ Department of EXTC \\ DJ Sanghvi College of \\ Engineering
}

Vile Parle (W) Mumbai, India

\begin{abstract}
The log periodic antenna and Yagi-Uda antenna are used in the applications where very high directivity is required. They also give very high gain in the range of $17-20 \mathrm{dBi}$. This paper presents a review on various configurations of log periodic and Yagi antennas, their advantages and problems. One problem encountered with Yagi-Uda antenna is relatively less bandwidth. This problem is solved by log periodic antenna which can operate over high bandwidth and providing high gain at the same time. In this paper, a review of various techniques to realize printed Yagi-Uda and log periodic antenna is discussed. They are realized by using different feeding techniques like microstrip feeding, co-axial feeding etc. They are also realized by using modifying the shape of directors and reflectors. The high bandwidth (log periodic antenna) has also been realized by increasing the number of reflectors.
\end{abstract}

\section{Keywords}

Yagi-Uda antenna, Log Periodic antenna, Printed Yagi-Uda antenna, Printed Log Periodic antenna.

\section{INTRODUCTION}

A Yagi antenna, also known as a Yagi-Uda antenna or a yagi, is a type of antenna array often used in TV antenna, to collect radio signals from space, and as a cellular antenna. Depending on its construction, it can be used for communications on frequencies from short wave to microwave, covering the spectrum from very high frequency (VHF) through ultra-high frequency (UHF). The Yagi antenna is a directional antenna, meaning its transmission and receiving power is focused in one direction and that it needs to be pointed in the specific direction one wants to receive or transmit. It is also high-gain, meaning it allows for increased signal strength both when receiving, by capturing more of the radio signal, and when transmitting, by allowing more of the transmitted power to be sent. It has moderate gain which depends on the number of elements used, typically limited to about $17 \mathrm{dBi}$, linear polarization, unidirectional (end-fire) beam pattern with high front-to-back ratio of up to $20 \mathrm{~dB}$ and is lightweight, inexpensive and simple to construct. The bandwidth is narrow, a few percent of the center frequency, and decreases with increasing gain, so it is often used in fixed-frequency applications.

The problem of less bandwidth is overcome by the log periodic antenna, which is able to provide directivity and gain while being able to operate over a wide bandwidth. In particular the log periodic dipole array is the most widely used version of this antenna family. The log periodic antenna is used in a number of applications where a wide bandwidth is required along with directivity and a modest level of gain. It is sometimes used on the HF portion of the spectrum where operation is required on a number of frequencies to enable communication to be maintained. It is also used at VHF and UHF for a variety of applications, including some uses as a television antenna. It has many similarities to the more familiar Yagi because it exhibits forward gain and has a significant front to back ratio. In addition to this the radiation pattern of this RF antenna design stays broadly the same over the whole of the operating band as do parameters like the radiation resistance and the standing wave ratio.

\section{LOG PERIODIC MSA}

The configuration shown in Fig. 1 is a log periodic antenna. The attainable gain can be up to $10 \mathrm{~dB}$ at a very small back lobe of up to $35 \mathrm{~dB}$. The base resistance is 50 to $120 \Omega$ depending on construction. The electrical qualities of such antennae recur periodically with the logarithm of the frequency. These periodical fluctuations of e.g. gain and base resistance can, however, be kept so small within a period by a suitable dimensioning of the antenna that one can consider her an approximation way constantly about the regarded frequency interval.

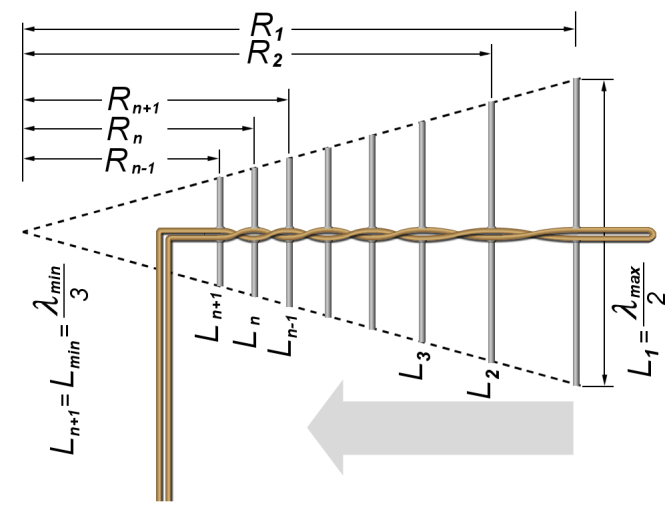

Fig.1: Log Periodic Antenna [1]

Here $\tau=\frac{L_{2}}{L_{1}}=\frac{L_{3}}{L_{2}}=\cdots=\frac{L_{n+1}}{L_{n}}=\frac{R_{2}}{R_{1}}=\frac{R_{3}}{R_{2}}=\cdots=\frac{R_{n+1}}{R_{n}} .$.

Where $\tau=$ Scale factor

$\mathrm{L}=$ Radiator length

$\mathrm{R}=$ Radiator distance

If the input impedance of a log-periodic antenna is plotted as a function of frequency, it will be repetitive. However, if it is plotted as a function of the logarithm of the frequency, it will be periodic (not necessarily sinusoidal) with each cycle being exactly identical to the preceding one. Hence the name logperiodic, because the variations are periodic with respect to the logarithm of the frequency. This repetition in frequency results in wide operating bandwidth. [1] A typical variation of the impedance as a function of frequency is shown in Fig. 2. 
Other parameters that undergo similar variations are the pattern, directivity, beamwidth, and side lobe level. [1]

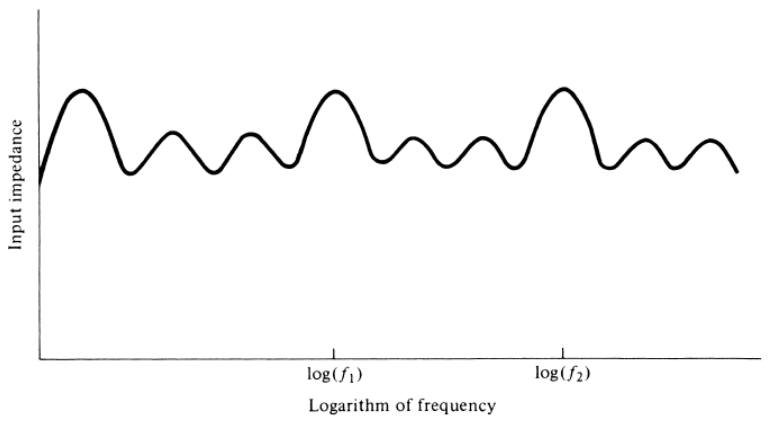

Fig.2: Typical input impedance variation of a log-periodic antenna as a function of the logarithm of the frequency [1]

\subsection{Printed Microstrip Log - Periodic}

\section{Antenna [2]}

The traditional structure of an MLP A excited by a pair of parallel lines (connection lines) is shown in Fig. 3. Along the center line is the connection lines that are used to be feed at the end of the shortest element by a coaxial feeder. The pairs of printed dipole elements are connected to the connection lines. All the adjacent dipole elements are printed on the two sides of the microstrip substrate, in an alternate way. The parameters of the MLPA, S, L and W are related to the scaling constant $\tau$ and are calculated using equation (1). The MLP A is made on FR4 with $\varepsilon=2.65$ and thickness $=1.5 \mathrm{~mm}$. Fig. 4 shows the measured reflection coefficient of the MLP A. From Fig. 4, it can be shown that the MLPA can work from $220 \mathrm{~Hz}$ to $6040 \mathrm{~Hz}$ with $S_{11}$ less than $-10 \mathrm{~dB}$. The relative bandwidth of the proposed antenna is found to be more than 97\%.

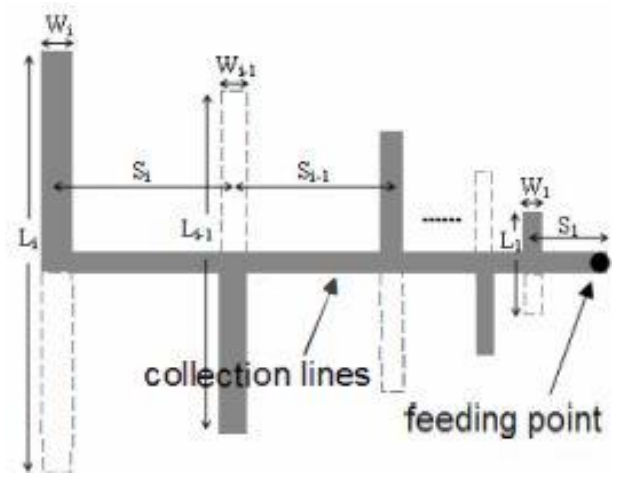

Fig.3: The traditional geometry of MLPA [2]

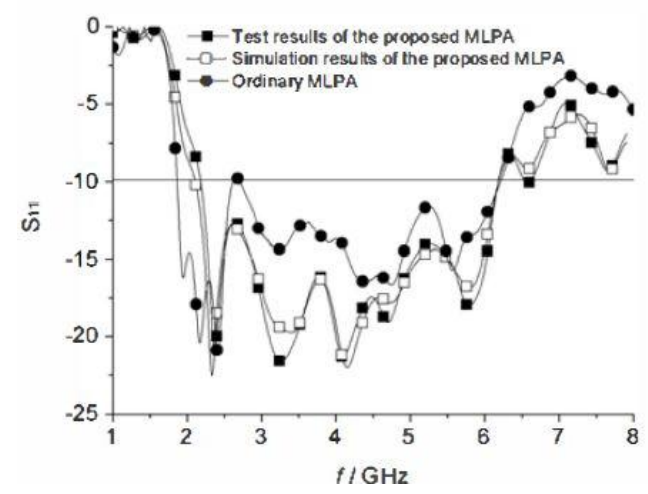

Fig.4: Reflection coefficient of MLPA [2]

\subsection{Design and Analysis of Five Element Microstrip Log-Periodic Antenna [3]}

The design principle for log-periodic antenna requires scaling of dimensions for period to period so that performance is periodic with the logarithm of frequency. This principle can be applied to an array of patch antennas. The patch length (L), the width $(\mathrm{W})$ and inset $(\mathrm{I})$ are related to the scale factor $(\tau)$. If all the dimensions of the array are multiplied by $\tau$, it scales into itself with element $n$ becoming element $n+1$, element $n+1$ becomes $\mathrm{n}+2$ and so on. The self-scaling property implies that the array will have the same radiating properties at all frequencies that are related by a factor of $\tau$. The various relations for square patch antenna is

$$
f_{r}=\frac{c}{2(L+2 \Delta L) \sqrt{\epsilon_{e f f}}}
$$

Where, $\mathrm{c}$ is the velocity of light.

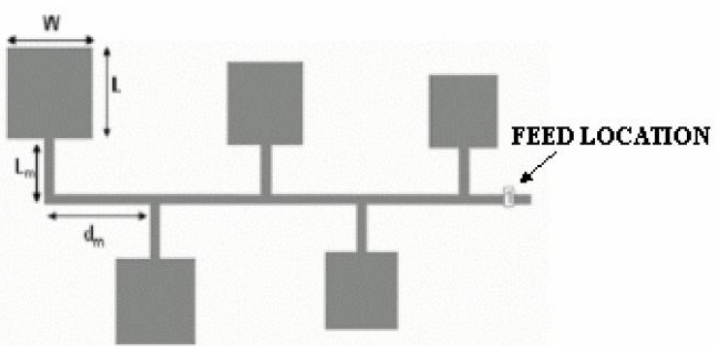

Fig.5: Proposed Geometry of Microstrip LPA [3]

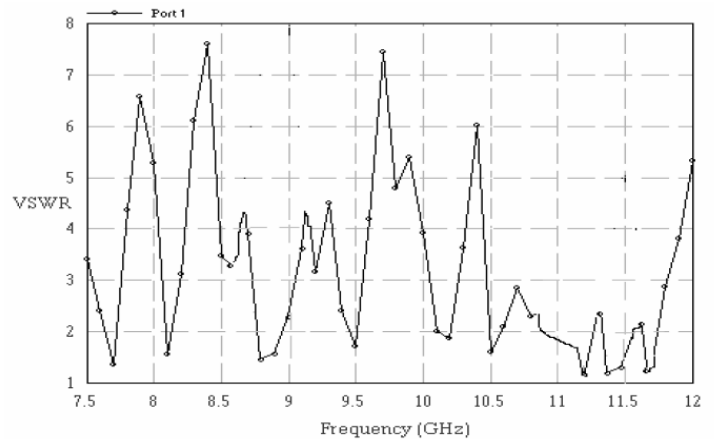

Fig.6: Frequency v/s VSWR [3]

The design of five element microstrip log-periodic antenna as shown in Fig. 5 is simulated on a method of moment based commercial software IE3D. The Fig.6 shows the graph between VSWR and Frequency. The antenna bandwidth for five elements is $11 \%$ from the center frequency at $11.2 \mathrm{GHz}$. The center frequency is defined here as $\left(f_{H}+f_{L}\right) / 2$ where $f_{H}$ and $\mathrm{f}_{\mathrm{L}}$ are the higher and lower $3 \mathrm{~dB}$ frequencies.

\subsection{Wideband simplified feed for printed log periodic dipole array antenna [4]}

The peak radiation of the printed log-periodic dipole array (PLPDA) antenna is fixed and the radiation pattern is stable within the working frequency, which leads to it being a good candidate for wideband efficient communication applications. In modern broadband communication systems, the wideband antenna should be indispensable with the linear phasefrequency function. However, the PLPDA antennas are mostly fed from the shorter end, and it introduces a nonlinear function of phase-frequency characteristics, which prevents their use for wideband communication. To solve this problem, the antenna can be fed from the longer, lower frequency end. However, the antenna structure is composed of two-double layer substrate, or it is fed by the CPW with an air-bridge. 
Therefore, those feeding structures increase the complexity and cost of the antenna.

However, when a simplified microstrip feed is used for a single layer substrate PLPDA antenna, it provides the advantages of low profile, broadband, a simple feeding network and ease of integration to the planar circuits. All the printed dipoles are cross-symmetrically spaced along the parallel feed lines on both sides of the dielectric substrate. The geometry of the PLPDA antenna is shown in Fig. 7. The top metallic layer is in Fig. 7(a) and the bottom one is in Fig. 7(b), respectively.

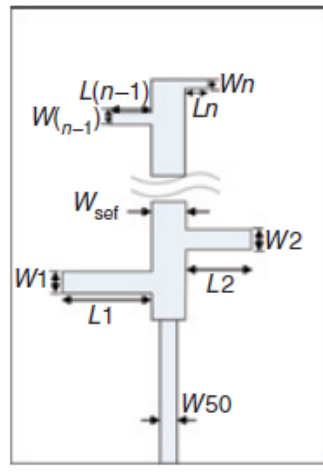

(a)

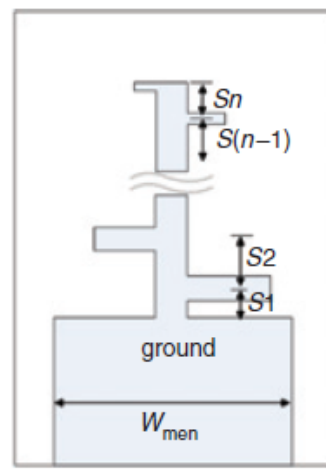

(b)
Fig.7: (a) Top layer, (b) Bottom layer [4]

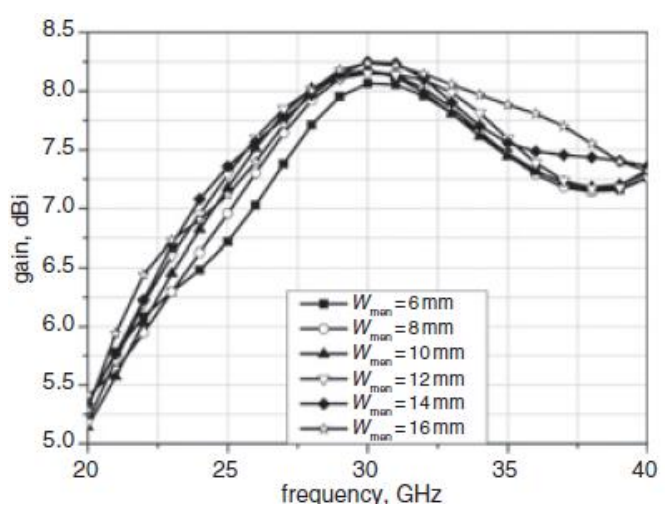

Fig.8: Simulated gain of proposed antenna with different $\mathbf{W}_{\text {men }}[4]$

Fig. 8 shows the simulated gain of the proposed antenna with different $\mathrm{W}_{\mathrm{men}}$. From the simulated results, as $\mathrm{W}_{\text {men }}$ increases, gain goes on increasing, particularly in higher frequency band. This increase in gain is less in lower frequency band. The increase of the gain depresses the microstrip ground that acts as the reflector. The detailed dimensions of the proposed antenna are:

$\mathrm{W}_{1}=1.21 \mathrm{~mm}, \mathrm{~W}_{2}=0.75 \mathrm{~mm}, \mathrm{~W}_{3}=0.46 \mathrm{~mm}, \mathrm{~W}_{4}=0.29$ $\mathrm{mm}, \mathrm{L}_{1}=1.95 \mathrm{~mm}, \mathrm{~L}_{2}=1.2 \mathrm{~mm}, \mathrm{~L}_{3}=0.75 \mathrm{~mm}, \mathrm{~L}_{4}=0.46$ $\mathrm{mm}, \mathrm{S}_{1}=1.8 \mathrm{~mm}, \mathrm{~S}_{2}=1.84 \mathrm{~mm}, \mathrm{~S}_{3}=1.14 \mathrm{~mm}, \mathrm{~S}_{4}=0.71$ $\mathrm{mm}$, Wsef $=1.6 \mathrm{~mm}$, Wmen $=8 \mathrm{~mm}$ and $\mathrm{W}_{50}=1.5 \mathrm{~mm}$.

\subsection{A Microstrip-Fed Log-Periodic Slot Array [5]}

Log-Periodic slot arrays offer the potential for broadband performance in addition to end-fire patterns whose hear polarization is perpendicular to the plane of the array. A printed microstrip fed log-periodic slot array featuring a common cavity for all slots as shown in fig.9. For antenna analysis, first evaluation of the equivalent series impedance of an isolated slot fed by an infinitely long microstrip line is done. The impedance of each slot is then concatenated with the transmission lines connecting the slots to yield an equivalent circuit description of the array from which the input impedance and slot voltages can be calculated and using them aperture fields are found out. A covered microstrip feed line is chosen due to its simplicity of construction and its ability to excite a slot etched in its ground plane.

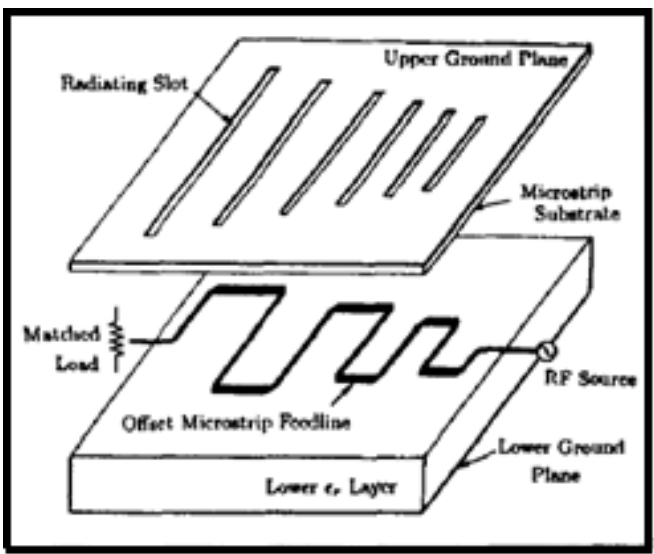

Fig.9: Geometry of the microstrip-fed log-periodic slot array [5]

Measurements were made on a ground plane of $12 \times 18$ inches with a 3 inch radius rolled edge in the backfire direction. Upper and lower ground planes were electrically connected all around the perimeter, thus forming an enclosed cavity. Inside this cavity, around its perimeter, were placed several pieces of absorber to suppress reflections of the dominant LSM mode. Fig.10 indicates a peak VSWR of 2.71:1 for the 500 to 2500 $\mathrm{MHz}$ frequency range. The predicted VSWR is also cyclic with a peak of $2.25: 1$ for the 1.0 to $2.0 \mathrm{GHz}$ range.

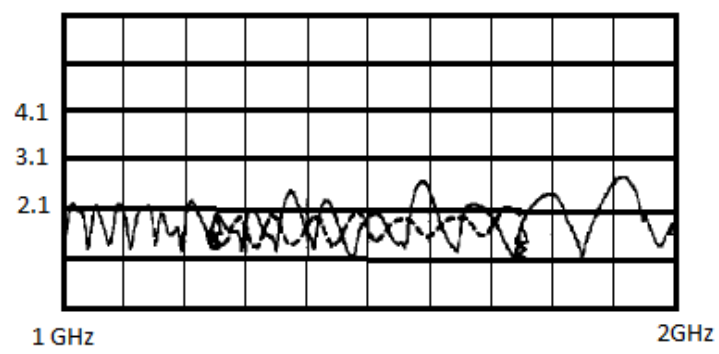

Fig.10: Predicted (dashed line) and Measured VSWR [5]

\subsection{A Comparative Study of Three Ultra Wideband Log-Periodic Microstrip} Antenna Arrays [6]

The structure of the designed antennas consist of two isotropic dielectric substrate layers: in the bottom layer a conventional microstrip line is separated from ground plane by dielectric substrate; in the upper layer the patch resonators are located and laterally displaced in alternate directions from the feed line. The low-cost fiberglass dielectric substrate FR-4 with the thickness $1.50 \mathrm{~mm}$ and dielectric constant 4.4 was used for the two dielectric layers. The width of the microstrip feed-line is equal to $2.87 \mathrm{~mm}$ for all designed antennas. The length of microstrip feed-line varies in accordance to the number of elements in the array. For the array with rectangular elements, the first element was designed with the following dimensions: $W=5.84 \mathrm{~mm}, L=7.24 \mathrm{~mm}$. The dimensions of the others rectangular elements can be obtained from equation (1) where 
the scale factor $\tau$ is equal to 1.05 . The same scale factor was used for all the others antennas presented in this paper. For the array of square patch elements, choice of side of the smallest square equal to $5.84 \mathrm{~mm}$ is made. For the array of circular patch elements was considered a diameter for the first patch element equal to $5.84 \mathrm{~mm}$. For all designed arrays the smallest spacing was keep constant and equal to $6.14 \mathrm{~mm}$. The choice of these dimensions result antenna arrays with the same total occupied area. The photograph presented in Fig. 11 shown three built log-periodic microstrip antenna prototypes with 15 patch elements.

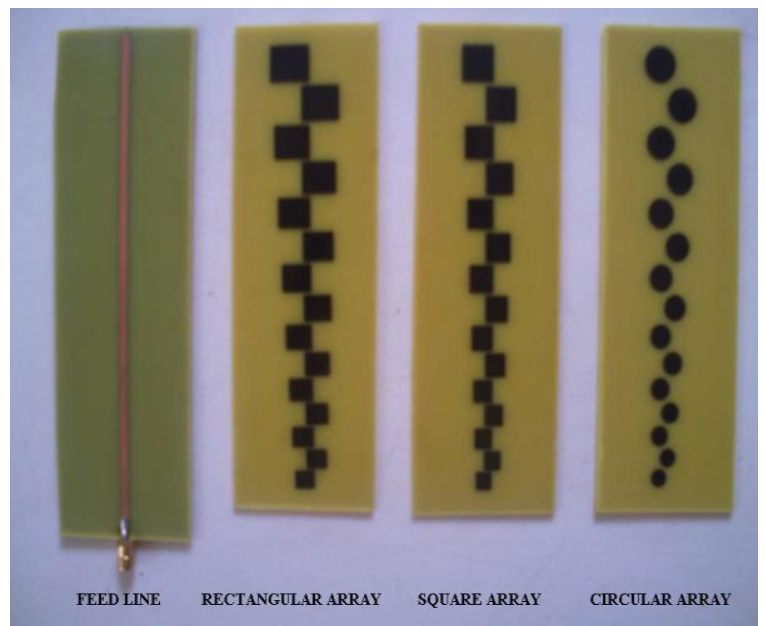

Fig.11: Built log-periodic microstrip antenna prototypes with 15 patch elements and total areas: Microstrip line is $160 \times 50 \mathrm{~mm}^{2}$, and patch element array is $150 \times 50 \mathrm{~mm}^{2}$ [6] Simulations were done using three different geometries (rectangular, square, and circular) and varying the number of elements: 5, 10 and 15 . The simulations were carried out in the range of frequencies of 1.0 up to $15.0 \mathrm{GHz}$. Fig. 12 compares the measure SWR for the built antennas.

It is verified that the number of elements of the array directly influences the array bandwidth performance. On the other side, the lower cut-off frequency of the antenna arrays depended on the choice of the patch geometries. Thereafter, in terms of $-10 \mathrm{~dB}$ return loss bandwidth, the rectangular patch presented the best performance, followed by the square and circular patch shapes, respectively.

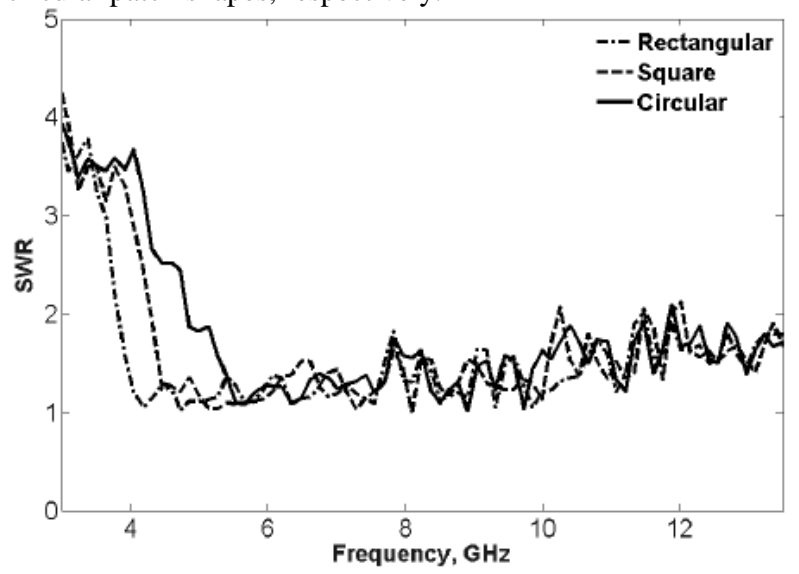

Fig.12 : Measured return loss considering for log-periodic microstrip antenna arrays with 15 patch elements [6]

\section{YAGI-UDA MSA}

A Yagi-Uda antenna, commonly known simply as a Yagi antenna, is a directional antenna consisting of multiple parallel dipole elements in a line, usually made of metal rods. It consists of a single driven element connected to the transmitter or receiver with a transmission line, and additional parasitic elements: a so-called reflector and one or more directors. The reflector element is slightly longer than the driven dipole, whereas the directors are a little shorter. The schematic diagram of Yagi-Uda antenna is shown in Fig.13. This design achieves a very substantial increase in the antenna's directionality and gain compared to a simple dipole.

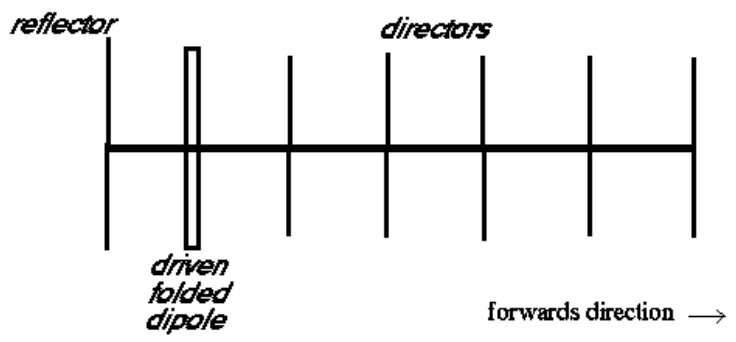

Fig.13: Schematic diagram of Yagi Uda Antenna

Only the driving element is connected directly to the feeder; the other elements couple to the transmitter power through the local electromagnetic fields which induce currents in them. The driving element is often a folded dipole, which by itself would have a driving point impedance of about $300 \mathrm{ohms}$ to the feeder; but this is reduced by the shunting effect of the other elements, so a typical Yagi-Uda has driving point impedance in the range $20-90 \mathrm{ohms}$.

\subsection{Low-Cost Yagi-Uda Monopole Array [7]}

A current potential application of Yagi Uda antennas is in GPRS (General Packet Radio Service), a packet-based data service over the existing GSM network. In general, the operation of mobile terminals require antennas with omnidirectional radiation patterns; however, in case the mobile terminal is far removed from the base station, a directional antenna must be used. Here, a new Yagi-Uda antenna topology, made up of printed monopoles, is studied. The geometry of the proposed Yagi-Uda antenna is shown in Fig.14. As noticed, it is made up of five printed monopoles: the driver element, a reflector, two directors, and a parasitic element positioned near the driver for increasing the antenna bandwidth. Since the monopoles are printed on both sides of a thin dielectric layer mounted perpendicularly to a ground plane, this new topology permits better electrical contact with the ground plane and greater design flexibility. Besides, as the monopole topology does not require a balun, the driver element can be fed directly via a SMA connector.

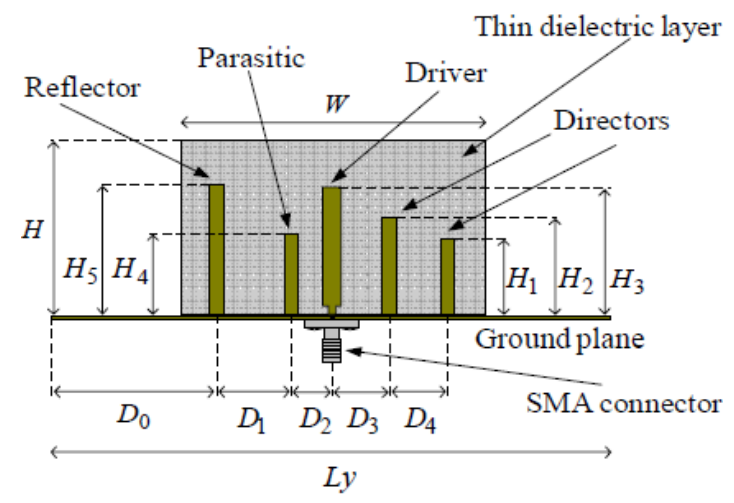

(a) 


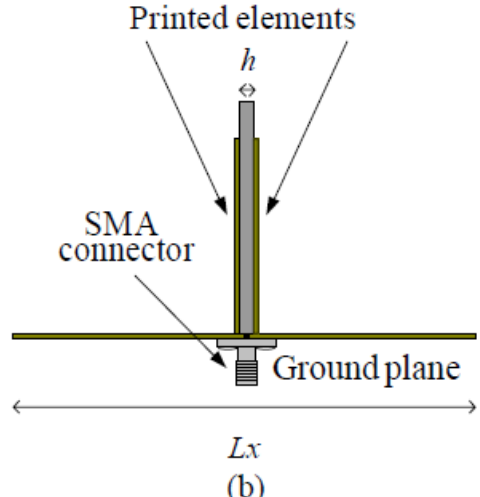

Fig 14: Yagi-Uda antenna composed of printed monopoles: (a) side view and (b) lateral view [7]

The proposed Yagi-Uda array was designed for broadband operation in the GPRS frequency range (Tx - 1.710 to 1.785 $\mathrm{GHz}$ and $\mathrm{Rx}-1.805$ to $1.885 \mathrm{GHz}$ ) with VSWR < 1.5 and $\mathrm{Zin}$ $=50 \Omega$. To keep costs low, a FR4 substrate (loss tangent $=$ 0.02 and $\varepsilon_{r}=4.4$ ) was used as the thin dielectric layer. After simulations performed with HFSS, experimental and simulated results for the VSWR of the prototype are compared in Fig.15. Good agreement is observed.

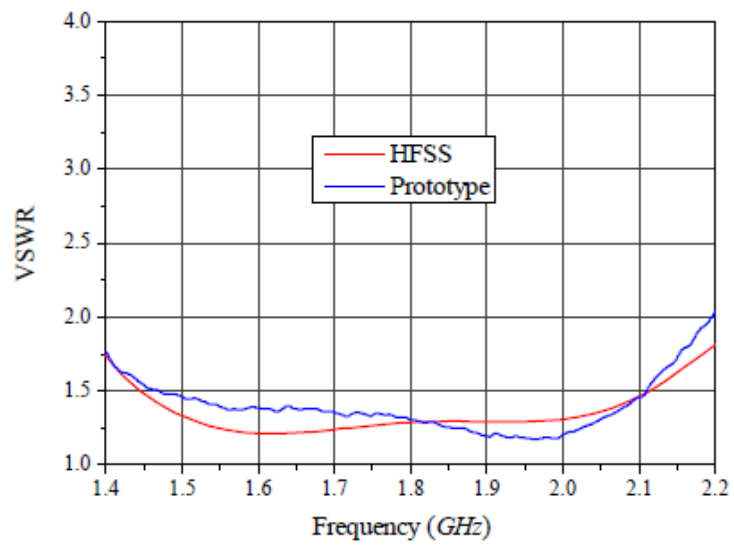

Fig 15: VSWR Curves [7]

Since the monopoles are printed on both sides of a thin dielectric layer mounted perpendicularly to a ground plane, this new topology permits better electrical contact with the ground plane and greater design flexibility. As a consequence, a compact, low cost, efficient radiator for a particular GPRS application was obtained.

\subsection{Broadband Printed-Circuit Yagi Array [8]}

The printed-circuit board (PCB) Yagi antenna has proven to be an effective radiator and an economical antenna with directional radiation property. The PCB Yagi has several advantages over traditional Yagi antennas, made of wired dipoles. However, the directional property of a Quasi-Yagi antenna is limited and the gain of a Quasi-Yagi is limited to about $5 \mathrm{dBi}$. Quasi-Yagi array is suit for utilization as a feed or radiation element of phased array. It is difficult to design a multi-element PCB Yagi with wider impedance bandwidth and simultaneously maintained broadband radiation characteristics as well as higher antenna gains. One way to accomplish the above mentioned broadband performance from a Yagi array is using a fat PCB dipole as driving element with the upper and lower quarter wavelength radiation elements having a fat stripe or wideband shape. The fat PCB dipole could be easily fed with a $50 \mathrm{ohm}$ micro-stripe line. It is well known that the currents on the Yagi radiation elements at all frequencies are evenly distributed from the centre of the dipole with very low current density approaching towards the dipole end. For fat dipoles, one can effectively picture that numerous semi-linear thin-line dipoles of varying lengths are effectively formed to excite multi-linear modes hence resulting in a very wide bandwidth.

Fig.16. shows a photograph and the basic dimensions of this seven elements PCB Yagi array antenna fabricated with dielectric constant 4.2. In its simplest configuration, this PCB Yagi consists of radiation elements having a fat dipole shape is designed to produce a directional patterns and broad bandwidth with linear polarization. The antenna consists of five isolated PCB dipole on the front as the directors, a pair of quarter wavelength arms on the front and back as the driving element, and the truncated ground plane on both the front and back sides as the reflector. The two quarter wavelength arms of the driving element are placed on the front and back sides of the substrate, a portion of the area is formed in the shape of a bridge to accommodate the quarter wavelength impedance transformer which is then connected to the $50 \mathrm{ohm}$ micro stripe feed line. The length of the first director is $25.32 \mathrm{~mm}$ and gradually tapered to $21.32 \mathrm{~mm}$ toward the director end.

To achieve the $43 \%$ designed impedance bandwidth, fat dipole radiators were etched on FR4 PCB with a thickness $0.762 \mathrm{~mm}$ and dielectric constant 4.2. For fat dipoles, one can effectively picture that numerous semi-linear thin-line dipoles of varying lengths are effectively formed to excite multi-linear modes hence resulting in a very wide bandwidth. With overall PCB size of $203 \mathrm{~mm} \times 65 \mathrm{~mm}$, this seven elements Yagi provides impedance bandwidth and suitable radiation patterns from $2.45 \mathrm{GHz}$ to $3.8 \mathrm{GHz}$.

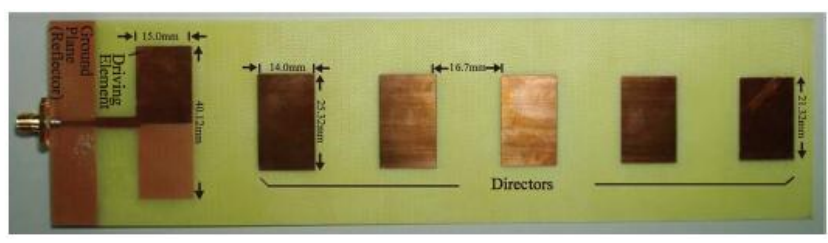

Fig. 16. Geometry of a seven elements, printed-circuit Yagi array etched on PCB with dielectric constant $\varepsilon_{r}=4.2$ [8]

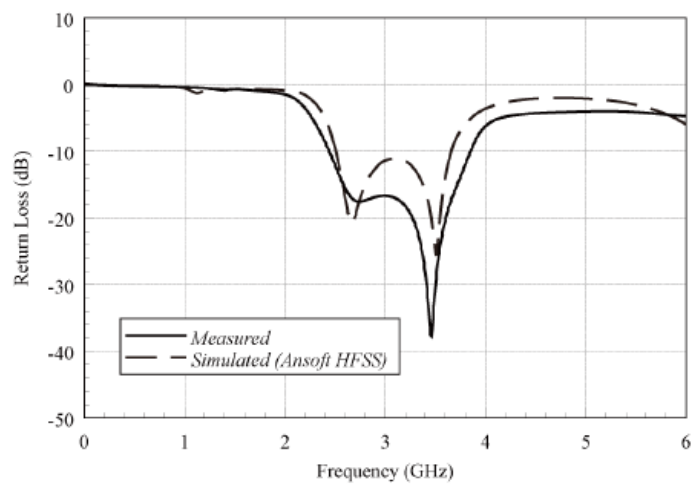

Fig. 17. Measured and simulated return loss of the seven elements Yagi array etched on FR4 with $\varepsilon_{r}=4.2[8]$

From Fig. 17, in the $2.45 \mathrm{GHz}$ to $3.8 \mathrm{GHz}$ range, the measured return loss is generally better than $-10.0 \mathrm{~dB}$. The return loss, simulated with IE3D software, shows in dotted line generally agrees with the return loss of the measured performance. With a proper choice of the dimensions of the array, a seven element Yagi etched on FR4 print-circuit board 
with dielectric constant 4.2 can provide a useful operating bandwidth from $2.45 \mathrm{GHz}$ to $3.80 \mathrm{GHz}$ for WiMax, WLAN, and Bluetooth wireless applications. By properly designing the number of directors and the dimensions of these radiating elements, antenna directivity from $8 \mathrm{dBi}$ to $11 \mathrm{dBi}$ can be easily achieved for $43 \%$ designed bandwidth.

\subsection{Yagi-Like Printed Antennas for Wireless Sensor Networks [9]}

The fast evolution of wireless communication systems has motivated a considerable increasing of the applications that make use of this technology. Many traditionally cable operated communication systems are migrating nowadays towards wireless technologies, due especially to the flexibility of this type of communication in the initial installation stage of these systems as well as on the subsequent extensions. Wireless sensor networks are also in the scope of this evolution, and more and more sensor networks supported by wireless systems are continuously implemented. However, the use of these wireless systems has an important disadvantage. Its small coverage (dozens of meters for Bluetooth and until about 200 meters for WI-FI) can considerably limit the deployment of the network. This limitation in the communication range is imposed by emitted power restrictions in this frequency band, associated to its open character and the potential dangerousness of the emission at these frequencies (close to the frequency of the domestic microwaves).

Here, two variations of printed antennas are presented, based on the well-known Yagi-Uda antenna, designed to cover the whole band of free use ISM. Both antennas, which have been named as Uniplanar Yagi Printed Antenna (Un-YPA) and Biplanar Yagi Printed Antenna (Bi-YPA), consists of a printed driven dipole and a series of additional elements in order to increase his directivity, in particular a reflector element and a certain number of director elements. The antennas have been designed onto a conventional low cost substrate in order to reduce the cost of manufacture. The antennas are analyzed and simulated by means of an in-house code, developed by the authors, based on the FDTD algorithm. The return losses (S11) have been calculated at the feed line of the antenna.

The design of both antennas is based on the traditional YagiUda antenna, widely used for long in all sort of applications where a moderate to high gain is needed (television, point to point communications). These antennas consist of a feed dipole, a reflector, which is usually a metallic stick or a metallic grid, and a series of elements parasites that operate as directors, so increasing the directivity and gain of the antenna

\subsubsection{Un-YPA}

The scheme of this antenna is drawn in Fig.18. The antenna displays two clearly differentiated parts. On one hand there is a feed zone, consisting of a $\lambda / 4$ impedance matching section and a phase shift network. The latter is implemented in order to properly adequate the signal coming from the coplanar feed line (CPS) to the driven dipole. This feeding circuit is implemented in microstrip technology. Here, advantage of the backplane of the substrate has been taken to act as the reflector element of the antenna. On the other hand, there is a series of elements disposed only on the top face of the substrate. These elements are: the CPS feeding line of the driven dipole, the printed dipole itself and a series of director elements, equal in size, included in order to increase the antenna gain. For the antenna design a standard low cost substrate $\left(\varepsilon_{r}=3.9, h=1.52 \mathrm{~mm}\right)$ is used.

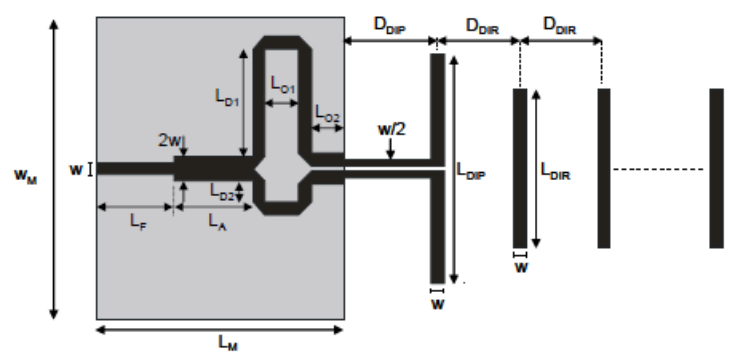

Fig. 18. Un-YPA scheme [9]

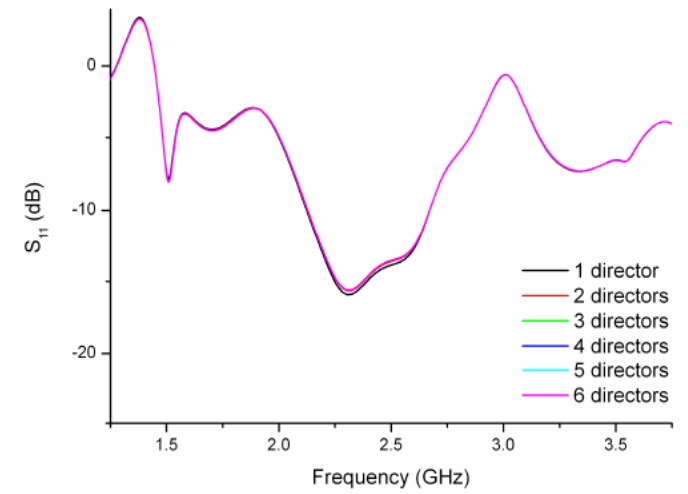

Fig. 19. Simulated return loss for the Un-YPA as a function of the number of directors [9]

The obtained results are shown in Fig.19 in the form of return loss. As observed, the number of director elements does not significantly influence on the antenna reflection behaviour.

\subsubsection{Bi-YPA}

In Fig.20 the scheme of the Bi-YPA is shown. The main difference observed with respect to the Un-YPA is on the printed driver dipole. In this later case each arm of the dipole is printed on each of the metallic faces of the dielectric substrate, respectively. With this configuration the suitable feed phase for the printed dipole is obtained by means of a couple of printed lines on both faces of the substrate, without the need of including an additional phase shifter. This kind feeding configuration reduces the size to a great extent, since the microstrip phase change as well as the $\lambda / 4$ impedance matching are eliminated. Also, the truncated ground backplane placed in the bottom face of the substrate is used as the reflector element. As observed in the scheme of this antenna, a series of equally sized director elements are placed on the top face of the substrate in order to increase the directivity of the antenna and, then, the gain.

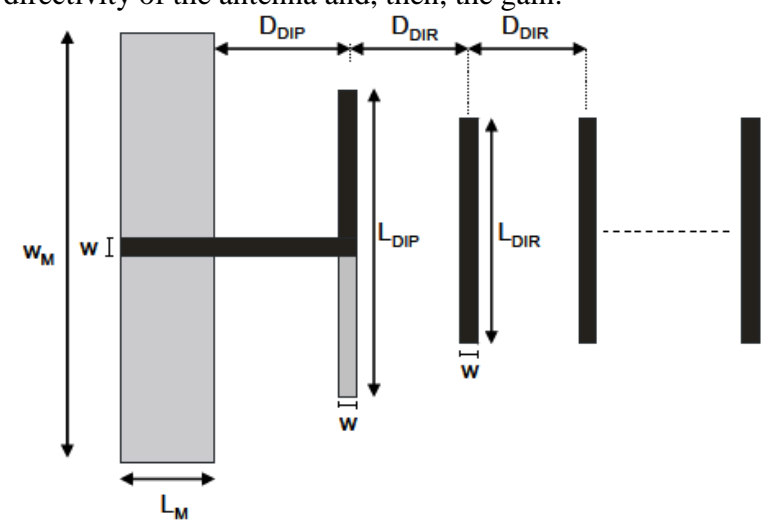

Fig. 20. Bi-YPA scheme [9] 


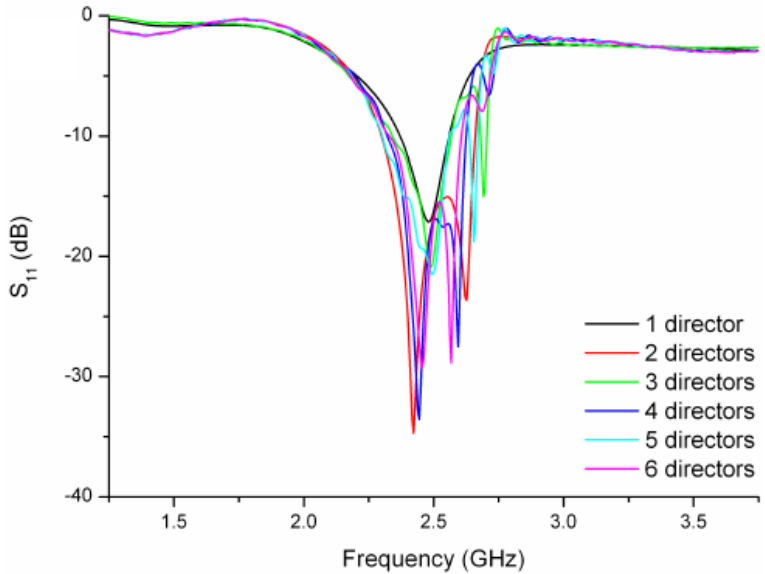

Fig. 21. Simulated return loss for the Bi-YPA as a function of the number of directors [9]

The used substrate in the antenna design was the same that for the Un-YPA (low cost substrate with $\varepsilon_{\mathrm{r}}=3.9$ and $\mathrm{h}=1.52 \mathrm{~mm}$ ). In Fig.21, the return losses are displayed. As observed, the number of director elements of the antenna slightly modifies the response of the S11 parameter. In any case, the central working frequency is kept practically constant, with a bandwidth around $10 \%$, adequate to cover the whole ISM band.

\section{CONCLUSION}

The Yagi antenna is a directional antenna, meaning its transmission and receiving power is focused in one direction and that it needs to be pointed in the specific direction one wants to receive or transmit. It is also high-gain, meaning it allows for increased signal strength both when receiving, by capturing more of the radio signal, and when transmitting, by allowing more of the transmitted power to be sent. The log periodic antenna is used in a number of applications where a wide bandwidth is required along with directivity and a modest level of gain. The frequency independent concept of $\log$ periodic antenna widens the bandwidth to a very great extent and thus this antenna can be used in the applications where wide bandwidth is desired. Depending on application, further implementation of log periodic and yagi antenna is to be done on microstrip patches, in order to make MSA's more directional antenna.

\section{REFERENCES}

[1] Antenna Theory, Analysis and Design by Constantine A. Balanis

[2] X. Ding, B.-Z. Wang, R. Zang, "Design and Realization of a Printed Microstrip Log-Periodic Antenna", IEEE, Electromagnetics; Applications and Student Innovation (iWEM), AUG 2012 IEEE International

[3] D. Sharma, R. Kumar, "Design and Analysis of Five Element Microstrip Log-Periodic Antenna", IEEE, Applications of Electromagnetism and Student Innovation Competition Awards (AEM2C), 2010, pp. 210-214

[4] G.H. Zhai, Y. Cheng, D. Min, S.Z. Zhu and J.J. Gao, "Wideband simplified feed for printed $\log$ periodic dipole array antenna", IEEE, Electronics Letters, Vol. 49, pp. $1430-1432$

[5] W. McKinzie, J. Moncada, T. Anderson, "A microstripfed log-periodic slot array", IEEE, Antennas and Propagation Society International Symposium, 1994. APS. Digest, Vol. 2, pp. 1278-1281

[6] V. Filho, P. Silva, A. D'Assunção, "A comparative study of three ultra-wideband log-periodic microstrip antenna arrays", IEEE, Antenna Technology (iWAT), 2010

[7] WorkshopD.C. Nascimento, R. Schildberg, and J. Lacava, "Low-Cost Yagi-Uda Monopole Array", Antennas and Propagation Society International Symposium, 2008. AP-S 2008. IEEE

[8] C. Lee, C. Lin, C. Tsai and C. Wang, "Broadband Printed-Circuit Yagi Array", Wireless Communications, Networking and Mobile Computing, 2009. WiCom '09

[9] E. Navarro, J. Blanes, J. Carrasco, C. Reig, "Yagi-Like Printed Antennas for Wireless Sensor Networks", IEEE, Sensor Technologies and Applications, OCT 2007 pp. 254-259R. 